\title{
Enhanced Detection Efficiency of Plastic Scintillators upon Incorporation of Zirconia Nanoparticles
}

\author{
Yuki Araya, Masanori Koshimizu*, Rie Haruki ${ }^{1}$, \\ Fumihiko Nishikido $^{2}$, Shunji Kishimoto ${ }^{1}$ and Keisuke Asai \\ Department of Applied Chemistry, Graduate School of Engineering, Tohoku University, \\ 6-6-07 Aoba, Aramaki, Aoba-ku, Sendai 980-8579, Japan \\ 'Institute of Materials Structure Science, High Energy Accelerator Research Organization, \\ 1-1 Oho, Tsukuba, Ibaraki 305-0801, Japan \\ ${ }^{2}$ Molecular Imaging Center, National Institute of Radiological Sciences, \\ Anagawa 4-9-1, Inage-ku, Chiba 263-8555, Japan
}

(Received August 22, 2014; accepted January 15, 2015)

Key words: plastic scintillators, nanoparticles, loaded scintillators, synchrotron radiation

We attempted to enhance the detection efficiency of plastic scintillators by incorporating zirconia nanoparticles up to $30 \mathrm{wt} \%$. The scintillators became opaque at higher concentrations because of Mie scattering. A higher nanoparticle concentration led to a higher detection efficiency, and the light yield of these scintillators was higher than or comparable to that of a commercial plastic scintillator, NE142. In addition, the time resolution of the scintillation detector equipped with each sample scintillator was approximately $0.5 \mathrm{~ns}$. These results indicate that the incorporation of nanoparticles is a versatile and effective approach to enhance the detection efficiency of plastic scintillators.

\section{Introduction}

Plastic scintillators have widely been used for applications that require an excellent time resolution or a short dead time..$^{(1,2)}$ One of the shortcomings of plastic scintillators is the low detection efficiency for high-energy photons. Because plastic scintillators comprise polymer matrices and several kinds of organic fluorescent molecules, the probability of interactions with high-energy photons is low owing to the small atomic numbers of the constituent elements (e.g., $\mathrm{C}, \mathrm{H}, \mathrm{O}$, and $\mathrm{N}$ ).

One effective approach to enhancing the detection efficiency of plastic scintillators is incorporating metal-organic complex molecules into polymer matrices. ${ }^{(3,4)}$ However, this method has a limitation: The metal-organic molecules have limited solubility in the

*Corresponding author: e-mail: koshi@qpc.che.tohoku.ac.jp 
polymer, which limits the incorporation concentration. In addition, the light yield of the plastic scintillators is severely degraded by the incorporation of such molecules because the molecules quench scintillation. ${ }^{(5)}$

In this paper, we report another approach to enhancing the detection efficiency of plastic scintillators, which is the incorporation of inorganic nanoparticles. The incorporation of nanoparticles enables the high-concentration loading of elements with large atomic numbers. In addition, the severe degradation of the light yield is avoided because the nanoparticles do not act as quenching centers. Furthermore, the transparency of the scintillator can be maintained by using particles with diameters less than $100 \mathrm{~nm}$. Moreover, the materials are expected to be robust to thermal or photodegradation by the incorporation of nanoparticles. ${ }^{(6,7)}$ Recently, we have reported the scintillation properties of plastic scintillators incorporated with Hf-Si oxide nanoparticles. ${ }^{(8)}$ In this research, we used zirconia nanoparticles because they are readily available from several suppliers and $\mathrm{Zr}$ has a relatively large atomic number of 40 . In this study, we focused on the detection of high-energy X-ray photons from synchrotron radiation and the scintillation properties of plastic scintillators containing up to $30 \mathrm{wt} \%$ zirconia nanoparticles. The resultant scintillation properties were compared with those of a commercial plastic scintillator, NE142 (loaded with $\mathrm{Pb}$ at $5 \mathrm{wt} \%$ ).

\section{Materials and Methods}

All the reagents were used as received. $2 \mathrm{~g}$ of polystyrene (Sigma-Aldrich, Japan; average molecular weight: 350000) was dissolved in $20 \mathrm{~g}$ of $\mathrm{N}, \mathrm{N}$-dimethylformamide (Wako, Japan), followed by the addition of a fluorescence molecule, i.e., 2-(4-tertbutylphenyl)-5-(4-biphenylyl)-1,3,4-oxadiazole (butyl-BPD; TCI, Japan). The amount of butyl-BPD was $0.5 \mathrm{~mol} \%$ relative to the number of monomer units of the polystyrene. Polystyrene was used as matrix because the energy transfer is efficient from the matrix to butyl-BPD. Zirconia nanoparticles with a diameter of $42 \mathrm{~nm}$ (Hosokawa Micron Co., Japan) were then added to the solution. The nanoparticles were supplied as a dry powder. The sample scintillators were obtained after solvent evaporation in aluminum containers at $333 \mathrm{~K}$ for $1 \mathrm{~d}$ and subsequently at $393 \mathrm{~K}$ for $2 \mathrm{~d}$.

Photoluminescence spectra were measured using a spectrofluorometer (F4500, Hitachi, Japan). X-ray-induced scintillation spectra were measured using an X-ray tube operating at $40 \mathrm{kV}$ and $40 \mathrm{~mA}$ and a charge-coupled device (CCD)-based detector. The $\mathrm{X}$-ray detection capability was characterized using synchrotron X-rays at BL-14A at the Photon Factory, KEK. The measuring system was the same as that employed in a previous study, ${ }^{(9)}$ and the X-ray energy was $67.4 \mathrm{keV}$. Single-bunch-mode operation was used, and the period of the successive X-ray pulses was $624 \mathrm{~ns}$. The beam spot was approximately $1 \mathrm{~mm}$ in diameter. The sample scintillators were attached to a photomultiplier tube (PMT; R7400P, Hamamatsu, Japan) with optical grease. The area of the sample scintillator was significantly larger than the spot size of the beam. Therefore, the detection efficiency does not depend on the area but on the thickness of the scintillators. To measure the pulse height spectra, the detection signal was amplified with a charge-sensitive amplifier (2005, Canberra, CT, US) and a main amplifier (572A, 
Ortec, TN, US); a histogram was obtained using a multichannel analyzer (MCA; 5004 Aptec, NY, US). The shaping time of the main amplifier was set at $0.5 \mu \mathrm{s}$. To estimate the scintillation light yield, the pulse height spectrum of a 5-mm-thick plastic scintillator, i.e., NE142 (OKEN Co., Ltd., Japan), ${ }^{(10)}$ was also measured for comparison. The detection efficiency was determined by comparing the counting rate of the current scintillation detector with that of a scintillation detector equipped with 5-mm-thick NaI:Tl. Time-resolution curves were obtained via the following setup: The detection signals were amplified with a fast preamplifier (VT120A, Ortec, TN, US), and the X-ray arrival timing signals were supplied from the accelerator. The timing of the signals was determined using a constant fraction discriminator (CFD, 935, Ortec, TN, US). The time difference between the signals was converted into a pulse height using a time-toamplitude converter (566, Ortec, TN, US), and the converted signal was accumulated to form a histogram using the MCA mentioned above.

\section{Results and Discussion}

Figure 1 shows a photograph of the sample scintillators containing different concentrations of zirconia nanoparticles. The sample scintillators became translucent and eventually opaque with increasing nanoparticle concentration, which is due to the Mie scattering of the incident light by the nanoparticles. Figure 2 shows an optical microscopic image of a cross section of the samples. In this image, the horizontal direction corresponds to the vertical direction during solvent evaporation. It is clearly evident that the nanoparticles are distributed inhomogenously, particularly in the vertical direction. In the measurements of scintillation properties in the studies, the bottom during the solvent evaporation was attached to the PMT.
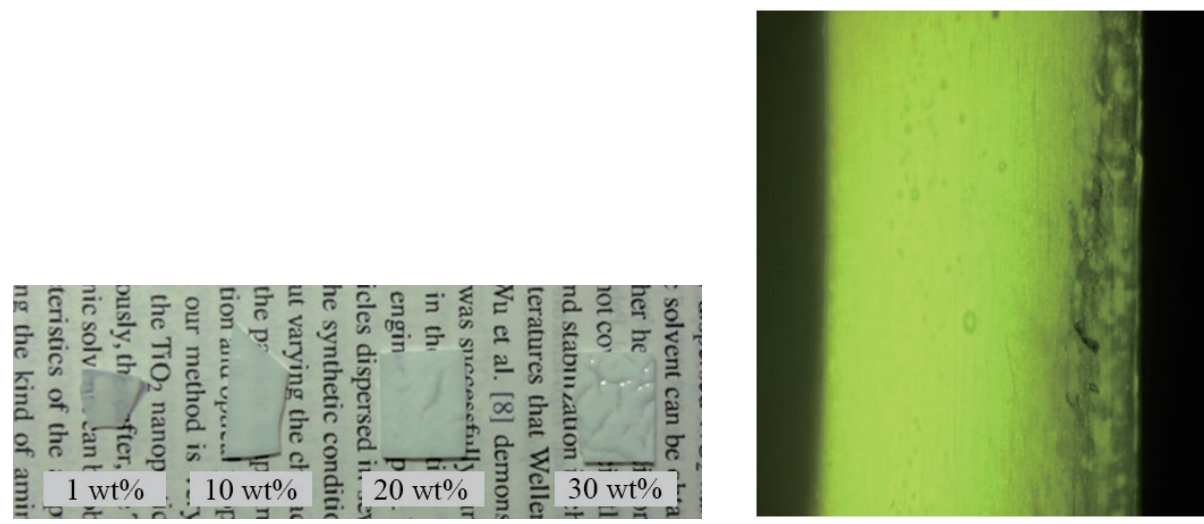

Fig. 1 (left). (Color online) Photograph of the sample scintillators containing different zirconia nanoparticle concentrations.

Fig. 2 (right). (Color online) Optical microscopic image of a cross section of the sample containing $30 \mathrm{wt} \%$ zirconia nanoparticles. The left-hand side corresponds to the bottom during the solvent evaporation. 
Figure 3 shows the photoluminescence spectra of the sample scintillators. The excitation wavelength was $305 \mathrm{~nm}$. The shapes and positions of the peaks in the spectra of the samples containing nanoparticles are similar to those of the sample without nanoparticles. The luminescence bands are ascribed to the fluorescence in butylBPD. The luminescence intensity seemingly increased with nanoparticle concentration. Because the butyl-BPD molecules are directly excited with an excitation wavelength of $305 \mathrm{~nm}$, this increase cannot be ascribed to the enhanced fluorescence quantum efficiency of the butyl-BPD molecules; this increase may be due to the Mie scattering of the luminescence photons. In the photoluminescence measurements, the sample surface was excited by the excitation light and fluorescence photons were detected from the surface. In this geometry, if we assume no contribution from the scattering of the fluorescent photons, we detect only the photons emitted from the butyl-BPD molecules in the direction of the photon detector. In the presence of Mie scattering, in addition to the fluorescent photons that directly reached the photon detector, some of the fluorescent photons emitted in the opposite direction were detected owing to Mie scattering within the sample. Thus, the detected luminescence intensity was higher for the samples containing nanoparticles. Figure 4 shows the X-ray-induced scintillation spectra. The emission wavelength was similar to that in the luminescence spectra.

Figure 5 shows spectra of the pulse heights of the scintillation detectors equipped with the sample scintillators for X-ray photons with $67.4 \mathrm{keV}$ energy. The incident X-ray fluence was the same for all spectra. It is clearly evident that the number of detection events increased with increasing nanoparticle concentration, which indicates that the detection efficiency was successfully enhanced by the incorporation of nanoparticles.

The pulse height is proportional to the number of photoelectrons at the PMT. The luminescence spectra of our scintillators and NE142 are similar. Thus, the sensitivity of the photocathode of the PMT is almost the same for our scintillators and NE142. In addition, the pulse height spectra were measured for the same X-ray photon energy. The pulse height is proportional to the number of photons emitted during one X-ray photon

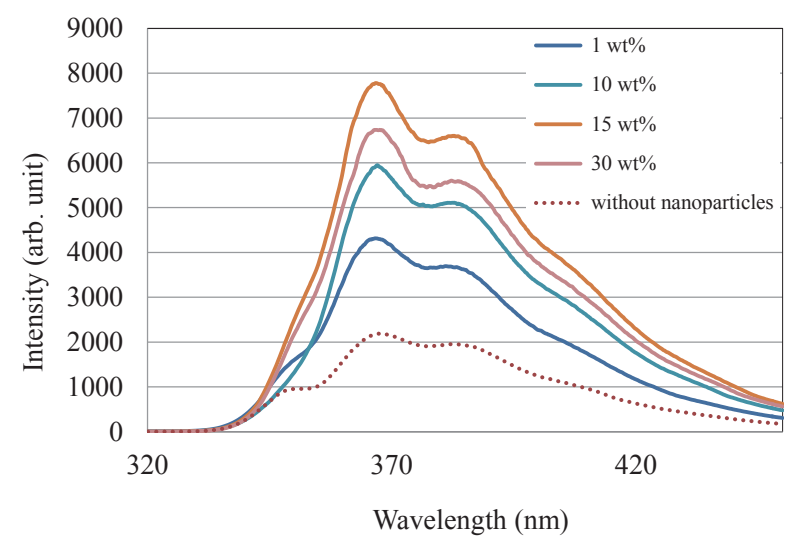

Fig. 3. (Color online) Photoluminescence spectra of the sample scintillators (excitation wavelength: $305 \mathrm{~nm}$ ). 


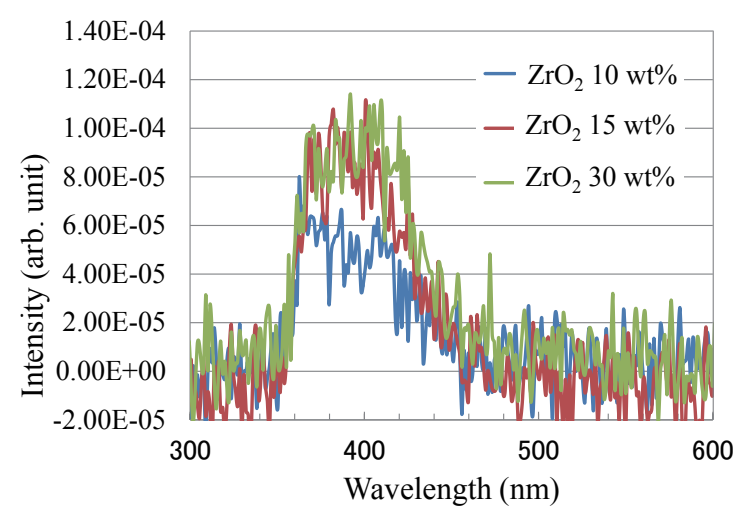

Fig. 4. (Color online) X-ray-induced scintillation spectra.

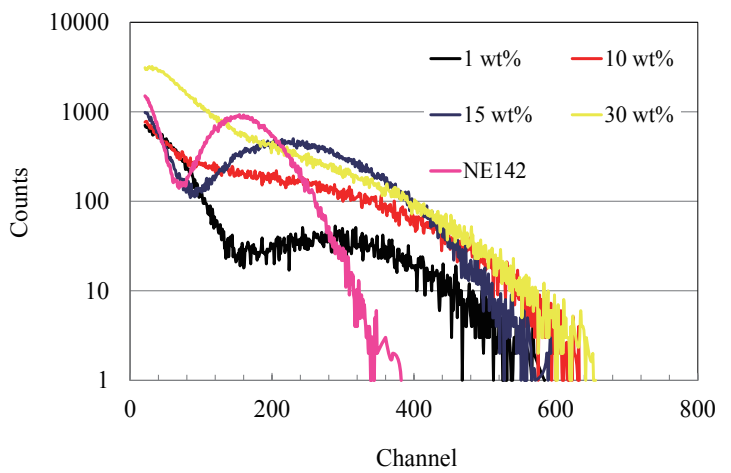

Fig. 5. (Color online) Pulse height spectra of the scintillation detectors equipped with the sample scintillators for $67.4 \mathrm{keV} \mathrm{X}$-ray photons.

detection event. Thus, the light yield was estimated by comparing the peak positions in the pulse height spectra. Peaks were observed at 380, 240, and 170 channels for the scintillator with 1 and $10 \mathrm{wt} \%$ nanoparticles and NE142, respectively. For the scintillator with $10 \mathrm{wt} \%$ nanoparticles, we observed a shoulder at approximately channel 350 . The light yield of the samples containing up to $10 \mathrm{wt} \%$ nanoparticles was higher than that of NE142. At higher nanoparticles concentrations, the photoelectron peak was relatively structureless, and the light yield was estimated to be comparable to that of NE142. The width of the photoelectron peak corresponds to the variation in the number of scintillation photons emitted during each detection event. A structureless photoelectron peak indicates a significant variation in the number of scintillation photons, which is due to the inhomogeneity of the nanoparticle distribution in the scintillators. Based on the high channel endpoint in the pulse height spectra, the light yield was not changed significantly by nanoparticle incorporation. This result indicates that the light yield was not severely degraded by the nanoparticle incorporation. 
The detection efficiencies of the sample scintillators are summarized in Table 1. The number of detection events is obtained as the total count in the pulse height spectrum. The number of detection events of the scintillation detector equipped with each scintillator is compared with that of a scintillation detector equipped with $\mathrm{NaI}: \mathrm{Tl}$ of $5 \mathrm{~mm}$ thickness for the same X-ray fluence. The detection efficiency was thus estimated assuming that the detection efficiency of the NaI:Tl scintillation detector was $100 \%$. It was clearly demonstrated that the detection efficiency is enhanced by incorporating nanoparticles up to $30 \mathrm{wt} \%$. Despite the significantly different thickness, we achieved a detection efficiency comparable to that of the 5-mm-thick NE142 for the sample scintillator containing $30 \mathrm{wt} \%$ nanoparticles.

Figure 6 shows the time-resolution curves of the scintillation detectors equipped with sample scintillators. A full width at half maximum (FWHM) time resolution of $\sim 0.5 \mathrm{~ns}$ was obtained for each scintillation detector, and no significant tail was observed. These results indicate that the short dead time of the plastic scintillators was maintained after the incorporation of the nanoparticles. Only the time-resolution curve for the $1 \mathrm{wt} \%$ nanoparticle scintillator had a long tail. Unfortunately, we cannot explain this difference at present.

Table 1

Efficiency of the detection of $67.4 \mathrm{keV}$ X-ray photons of each sample scintillator.

\begin{tabular}{lllllllc}
\hline $\begin{array}{l}\text { Nanoparticle } \\
\text { concentration [wt\%] }\end{array}$ & 1 & 5 & 10 & 15 & 20 & 30 & NE142 \\
$\begin{array}{l}\text { Detection efficiency [\%] } \\
\text { Thickness [mm] }\end{array}$ & 1.1 & 1.3 & 2.1 & 3.1 & 5.7 & 7.4 & 10.7 \\
\hline
\end{tabular}

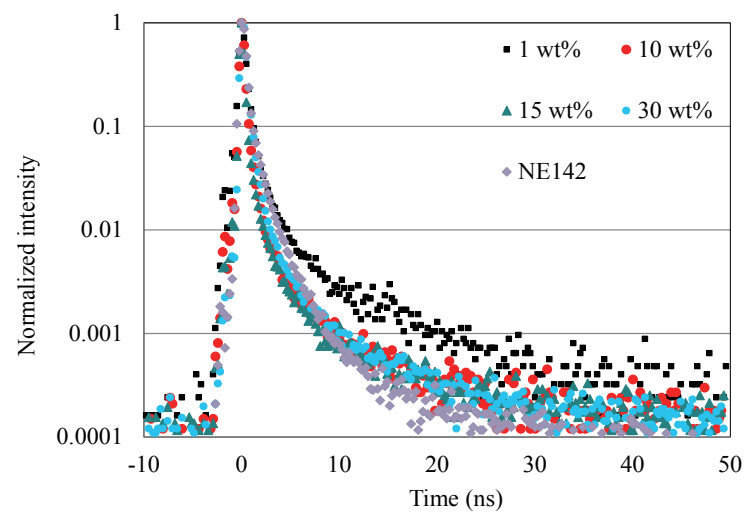

Fig. 6. (Color online) Time-resolution curves of the scintillation detectors equipped with sample scintillators. 


\section{Conclusion}

We synthesized plastic scintillators containing inorganic nanoparticles in order to enhance the detection efficiency for high-energy photons. The light yields of the samples were higher than or comparable to that of a commercial plastic scintillator, i.e., NE142. The detection efficiency was successfully enhanced by the incorporation of zirconia nanoparticles, and the fast response of the plastic scintillators was maintained. These results indicate that the incorporation of nanoparticles is an effective and versatile method of enhancing the detection efficiency of plastic scintillators.

\section{Acknowledgements}

This research was partially supported by a Grant-in-Aid for Young Scientists (A) (No. 25709088, 2013-2015) and a Grant-in-Aid for Adaptable and Seamless Technology Transfer Program through Target Drive R\&D from the Japan Science and Technology Agency. It was performed with the approval of the Photon Factory Advisory Committee (Proposal Nos. 2008G104, 2010G179, and 2012G062). This work was partially supported by the SPRITS program, Kyoto University, and the Cooperative Research Project of Research Institute of Electronics, Shizuoka University.

\section{References}

1 J. L. Radtke: IEEE Trans. Nucl. Sci. 37 (1990) 129.

2 W. W. Moses and S. E. Derenzo: Nucl. Instrum. Methods Phys. Res., Sect. A 291 (1990) 435.

3 J. Dannin, S. R. Sandler and B. Baum: Int. J. Appl. Radiat. Isotopes 16 (1965) 589.

4 B. L. Rupert, N. J. Cherepy, B. W. Sturm, R. D. Sanner and S. A. Payne: EPL 97 (2012) 22002.

5 M. Hamel, G. Turk, S. Darbon and S. Normand: IEEE Trans. Nucl. Sci. 59 (2012) 1268.

6 T. Otsuka and Y. Chujo: Polym. J. 42 (2010) 58.

7 T. Otsuka and Y. Chujo: J. Mater. Chem. 20 (2010) 10688.

8 Y. Sun, M. Koshimizu, N. Yahaba, F. Nishikido, S. Kishimoto, R. Haruki and K.Asai: Appl. Phys. Lett. 104 (2014) 174104.

9 S. Kishimoto, K. Shibuya, F. Nishikido, M. Koshimizu, R. Haruki and Y. Yoda: Appl. Phys. Lett. 93 (2008) 261901.

10 Data sheet of BC452 from Saint-Gobain Ceramics \& Plastics Incorporated (BC452 is equivalent to NE142). 